\title{
Biofactors in food promote health by enhancing mitochondrial function
}

by Sonia F. Shenoy, Winyoo Chowanadisai, Edward Sharman, Carl L. Keen, Jiankang Liu and Robert B. Rucker

Mitochondrial function has been linked to protection from and symptom reduction in chronic diseases such as heart disease, diabetes and metabolic syndrome. We review a number of phytochemicals and biofactors that influence mitochondrial function and oxidative metabolism. These include resveratrol found in grapes; several plant-derived flavonoids (quercetin, epicatechin, catechin and procyanidins); and two tyrosine-derived quinones, hydroxytyrosol in olive oil and pyrroloquinoline quinone, a minor but ubiquitous component of plant and animal tissues. In plants, these biofactors serve as pigments, phytoalexins or growth factors. In animals, positive nutritional and physiological attributes have been established for each, particularly with respect to their ability to affect energy metabolism, cell signaling and mitochondrial function.

$\mathrm{O}$ ne of the most promising current areas of nutritional research focuses on plant compounds with positive health effects that extend beyond the functions of well-recognized essential vitamins, minerals and macronutrients (Rice-Evans and Packer 2003). Identifying such compounds and studying their mechanisms of action have been important activities of the UC Davis Center for Health and Nutrition Research (CHNR) (table 1).

Many of the human health-related biofactors in plants (e.g., various pigments, secondary metabolites and phytoalexins) have evolved to provide protective camouflage, repel predators or facilitate the transformation of specific wavelengths of light into chemical energy. Our food exposes us to thousands of such "xenobiotic" compounds (external chemicals
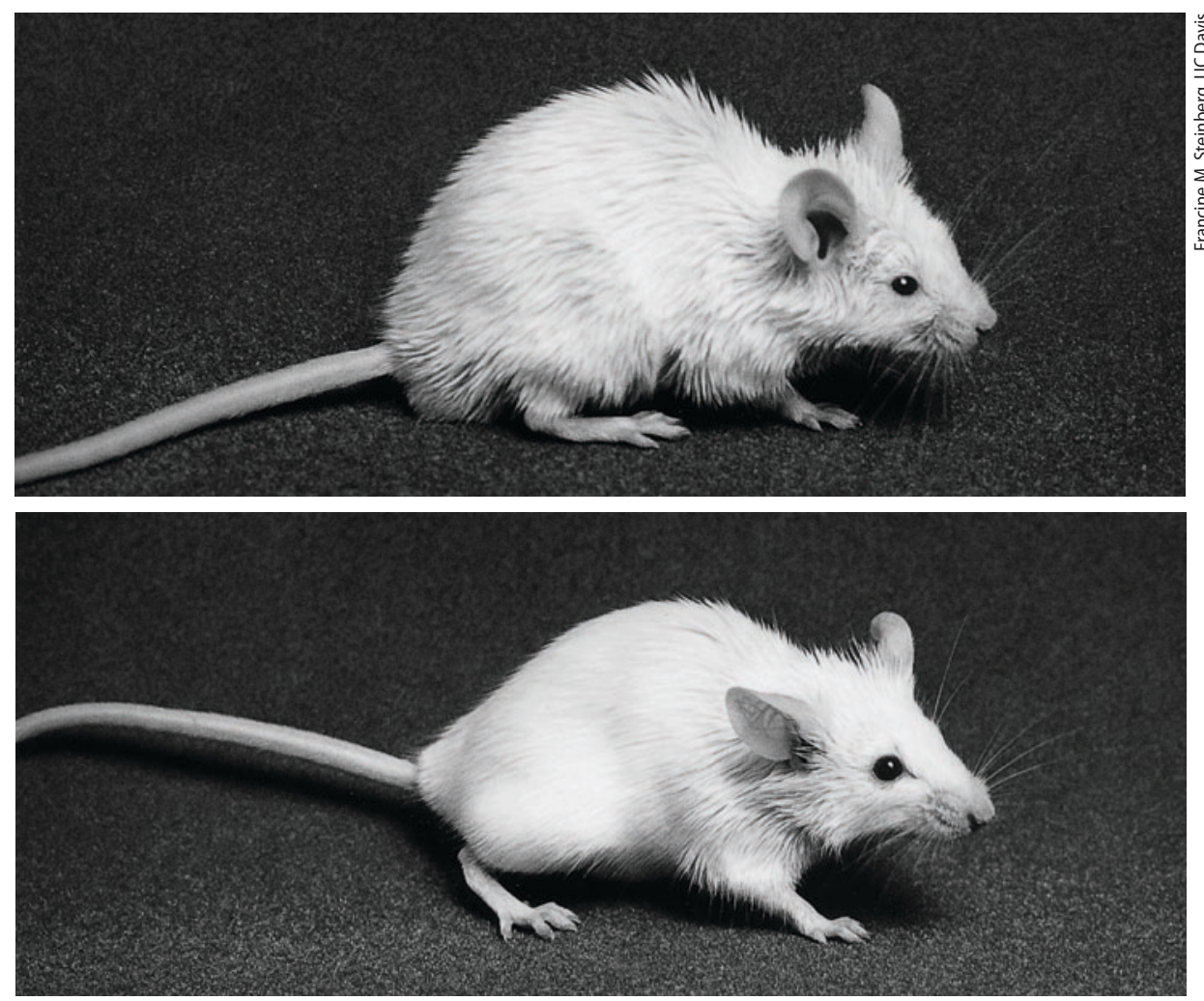

Biofactors in food play a role in enhancing mitochondrial function, thereby decreasing the risk of some chronic diseases. Top, a mouse that has been deprived of pyrroloquinoline quinone (PQQ), a ubiquitous bacterial compound found in fermented products, tea, cocoa and legumes. Above, a mouse fed a diet containing PQQ.

that our body does not normally produce) that must be either eliminated or put to novel uses in the body. Many xenobiotics in foods can influence specific metabolic functions, acting as bioactive factors (biofactors). For example, epidemiological studies have shown a correlation between foods high in bioactive factors such as flavonoids and the decreased risk of chronic diseases such as vascular disease and gastrointestinal tract cancers (Rice-Evans and Packer 2003; Wallace 2011). While we still have only a rudimentary understanding of how these bioactive compounds work, they can have profound and often specific effects on mitochondria (see page 136).

Biofactors in food that enhance mitochondrial function include resveratrol, quercetin, procyanidins, catechins, hydroxytyrosol and pyrroloquinoline quinone (fig. 1). Although it is easy to overstate mitochondria-related health claims, a broad range of healthful attributes has been described and validated for each of these compounds.

\section{Biological properties of resveratrol}

Resveratrol is a stilbenoid (a type of natural polyphenolic compound) and a phytoalexin, a class of compounds produced by some plants when under attack by pathogens such as bacteria or fungi. It is found predominately in purple grapes and juice, red wine, peanuts and some berries (Xia et al. 2010). In animals, resveratrol also has potent biological properties that have been reported to range from cardio-protection to enhanced neuronal activity. As examples, resveratrol exposure has been associated with longer life spans in yeast and in short-lived invertebrates, such as Caenorhabditis elegans

Online: http://californiaagriculture.ucanr.org/ landingpage.cfm?article=ca.v065n03p141\&fulltext=yes DOI: 10.3733/ca.v065n03p141 
(a type of roundworm) and Drosophila (a genus of small fruit flies), which are often used as experimental models in aging studies (Bass et al. 2007). It has been proposed that the effect of resveratrol on lifespan in invertebrate models is due to the induction of phase 2 drug detoxification or the activation of AMP kinase (one of the signaling molecules important to mitochondriogenesis) (see page 136).

However, in more complex vertebrate animal models, the effects of resveratrol on longevity can be variable (Agarwal and Baur 2011). Resveratrol has little effect on the longevity of lean mice or mice whose caloric intake is restricted, but it

TABLE 1. Recent Center for Health and Nutrition Research publications examining plant phytochemicals

\begin{tabular}{ll}
\hline \hline Phytonutrient & Publications \\
\hline Pyrroloquinoline quinone & Chowanadisai et al. 2010; Rucker et al. 2009; Tchaparian et al. 2010 \\
Epicatechins & Schroeter et al. 2006 \\
Cocoa flavanols & Balzer et al. 2008; Keen et al. 2005; Lanoue et al. 2010; Ottaviani et al. 2011 \\
Cocoa procyanidins & Keen et al. 2005 \\
Lichee flavanols & Kalgaonkar et al. 2010 \\
Hydroxytyrosol & Hao et al. 2010; Zhu et al. 2010 \\
Monounsaturated fats & Rajaram et al. 2009 \\
\hline
\end{tabular}

does influence the expression of several genes associated with longevity (e.g., sirtuins) (Baur 2010). Dietary supplementation with resveratrol in mice appears to prevent age-related declines in cardiovascular function and can improve the ability of mice to respond to inflammation (Baur 2010; Baur et al. 2006). Moreover, treating mice with resveratrol significantly increases their aerobic capacity, as evidenced by increased treadmill running time and muscle fiber oxygen consumption, and improved mitochondrial function (Baur et al. 2006; Murase et al. 2009). Mice treated with resveratrol also accommodate better than nontreated mice to oxidative stresses induced by exposure to various chemical agents (Kovacic and Somanathan 2010).

Relatively little is known about resveratrol's effects in humans. Interest in exploring this compound's potential health benefits increased after speculation that

\section{Glossary}

Acrolein (propenal): A chemical that can play a role in the balance between cell proliferation and apoptosis. Acrolein has been used as a toxicant in experimental models to study age-related macular degeneration and neurological diseases.

Amino acids: Nitrogen-containing compounds that, when linked together, form proteins or function independently as precursors to chemical messengers and as intermediates in metabolism. The body cannot make some amino acids in sufficient quantities, making them essential in the diet.

AMP kinase (AMPK): An enzyme involved in regulating cellular energy homeostasis. Upregulation of AMPK results in a decrease in energy consumption pathways coupled with the creation of ATP or cellular energy.

Anti-inflammatory: A compound (drug or food substance) that acts to reduce inflammatory processes or responses to injury or infection. Potential markers of an anti-inflammatory response include changes in the levels of high-sensitivity C-reactive protein or various cytokines, intercellular mediator in the generation of an immune response.

Bioavailability: Measure of the ability for a particular tissue to absorb and utilize a nutrient. Factors potentially affecting bioavailability include the food matrix, chemical form or current nutritional status.

Cytochrome c oxidase: A mitochondrial protein involved in energy metabolism via the transfer of electrons derived from the oxidation of given substrates.

Epidemiological: Large-scale observational studies that provide relationships (e.g., correlations) between experimental variables and related factors (e.g., food and health).
Flavanol (a class of flavonoids): Contain multiple phenol groups in a specific configuration that is the chemical basis for antioxidant potential. Flavanols are different from flavonols; flavanols are building blocks for proanthocyanidins, which include many compounds and their polymers, such as epicatechin, catechin and tannins. Examples of flavanol-rich foods include tea, cocoa and grapes.

Flavonol (another class of flavonoids): Also contain multiple phenol groups in configurations that are the chemical basis for antioxidant potential. They differ from flavanols in part due to their 3-hydroxyflavone backbone. Examples of flavonol-rich foods include cranberries and onions.

Monomers, oligomers, polymers: A monomer is the fundamental chemical unit of a compound, which is capable of linking together to form polymeric chains. An oligomer is a molecule that consists of a specifiable number of monomers (usually less than five). Unlike a polymer, if one monomer is removed from an oligomer, its chemical properties are altered. A polymer is repeating units of a monomer, usually resulting in compounds of high molecular weight. For the epicatechin and catechins, extensive polymerization results in tannin formation.

mtDNA (mitochondrial DNA): Genetic code (DNA) located in the mitochondria.

Phytochemicals, phytonutrients: Plant compounds that may have health-protecting qualities such as antioxidant activity or immune system enhancement.

Proanthocyanidins (procyanidin). Polymer chains of flavanols, such as epicatechins and catechins. Proanthocyanidins have little color, whereas the monomer units of anthocyanidins are usually red. 
resveratrol in wine could help explain the so-called French Paradox: Why the French seem to have a low incidence of coro-

nary heart disease, despite diets that are relatively rich in saturated fats (Kopp 1998).

Daily dosage. Given the intense interest in resveratrol, surprisingly few human clinical trials have been conducted. However, it is known that a daily dose in the range of 300 to 3,000 milligrams is required to achieve a significant clinical response in people (such as bringing high levels of blood glucose back to normal) (Elliott and Jirousek 2008). About the same amounts of resveratrol are required for animals, relative to the unit of dietary energy utilized (when expressed as milligrams resveratrol per kilocalorie). For example, in mice genetically modified to express type 2 diabetes, normalizing blood glucose and plasma insulin requires 100 to 500 milligrams resveratrol per 1,000 kilocalories. Significant clinical responses in humans also require resveratrol in this range, about 200 to 800 milligrams per day when typical caloric intakes of 2,000 to 2,500 kilocalories are consumed.

Food sources. The best food sources of resveratrol supply far less than the amounts required for significant clinical responses. For example, red wine, a major dietary source, seldom has more than 10 milligrams per liter, which raises questions about resveratrol-related health benefits based on wine consumption alone. As a consequence, researchers are currently pursuing questions related to resveratrol's bioavailability (ability of the body to absorb a substance).

Derivatives. Another area of active investigation is whether or not a derivative of resveratrol is more responsible than resveratrol itself for a given effect. Like other bioactive factors, once in the body resveratrol undergoes a number of metabolic transformations associated with fermentation, digestion and xenobiotic metabolism (see page 136). For resveratrol, the most abundant metabolites are resveratrol-3-O-glucuronide and resveratrol-3sulfate (Xia et al. 2010).

Studies designed to elucidate resveratrol's actions have nevertheless been useful as research templates for the now hundreds of studies dealing with the role and function of biofactors in general. For example, it has been reasonable to ask whether given flavonoids, flavanols or other types of phenolic compounds provide cardiovascular or neurological protection by mechanisms similar or dissimilar to those for resveratrol. A principle example is studies on resveratrol's modes of action as they relate to the signaling important to mitochondriogenesis.

\section{Quercetin and mitochondriogenesis}

Quercetin is a flavonol (a subclass of flavonoid) widely present in fruits and vegetables, particularly onions and apples. Depending on one's food choices, the daily estimated intake of

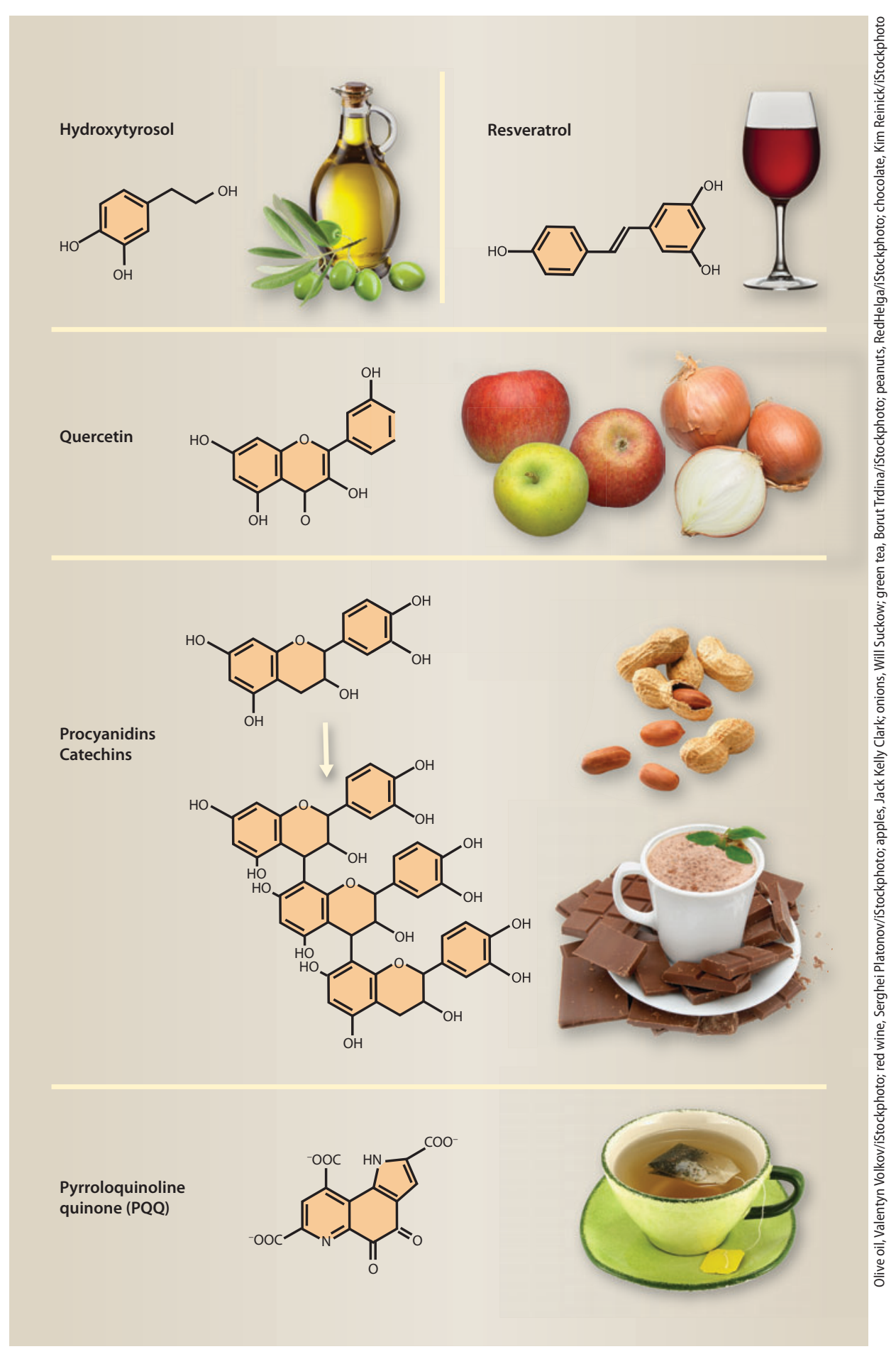

Fig. 1. Parent chemical structures of biofactors in food, and common sources. Derivatives may also be present, created by food processing or as a result of xenobiotic metabolism (see fig. 2). All of the compounds highlighted are polyphenolic (with multiple aromatic-OH groups). Quercetin and procyanidins are examples of flavonols and flavanols, respectively. 
quercetin ranges from 10 to 25 milligrams per day.

Mouse studies. Like resveratrol, quercetin has also been shown to induce mitochondrial biogenesis in mice (Davis et al. 2009, 2010). Mice fed quercetin have increased mitochondrial DNA, cytochrome c oxidase activity (a measure of mitochondriogenesis) and proteins key to mitochodriogenesis in both muscle and brain. Like resveratrol studies, these observations are supported by evidence of increased exercise tolerance following quercetin administration in mice.

Human studies. In humans, doses of quercetin similar to those for resveratrol are required to achieve an effect. In typical studies, usually 500 to 1,000 milligrams per day are given to elicit responses such as improvements in antiinflammatory or antioxidant activity and mitochondrial-related functions (Davis et al. 2009). Quercetin also accumulates in mice and rat mitochondria, an observation consistent with reports that it can act as a protector of mitochondrial function. In humans, only short-term quercetin trials have been performed. In one study, 500 milligrams of quercetin twice per day for 7 days caused a modest but significant increase in endurance capacity and $\mathrm{VO}_{2 \text { max }}$, the maximum oxygen uptake during aerobic exercise (Davis et al. 2010). The 7 days of quercetin feeding was associated with an increase in $\mathrm{VO}_{2 \max }$ (about $4 \%$ ) along with a $13 \%$ increase in bicycle ride time to fatigue (Davis et al. 2010).

For perspective, elite endurance athletes typically have a high $\mathrm{VO}_{2 \max }$. Training has been shown to increase $\mathrm{VO}_{2 \max }$ up to $20 \%$ from an individual's baseline; thus a $4 \%$ increase from merely taking a supplement is an intriguing prospect. In competitive situations, even small differences may be of importance. In other studies, particularly those involving nontrained subjects, quercetin had little or no significant effect on endurance-related parameters (Curetin et al. 2009; Dumke et al. 2009).

Quercetin is readily absorbed from the gut, and absorption is dose-dependent in humans (Hollman et al. 1995). It has been estimated that around half the quercetin in onions is absorbed, along with appreciable amounts of important quercetin derivatives such as quercetin-3-rutinoside and quercetin aglycone. A single dose of quercetin leads to a rise in blood plasma levels of this biofactor, which peak about 2 to 6 hours after ingestion and gradually decline over a $24-$ to 48 -hour period. This pattern of absorption is also typical of other members of the flavonoid family.

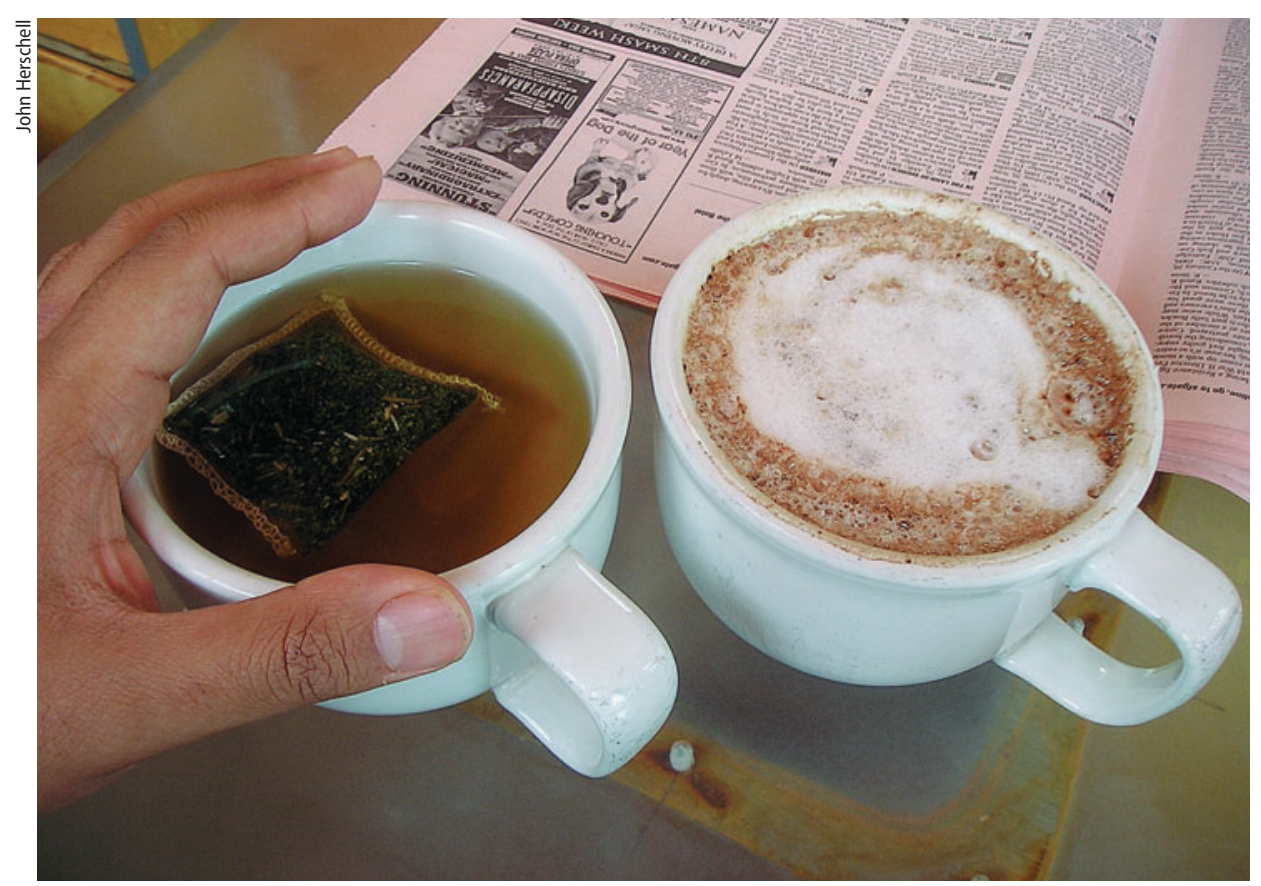

Flavanols are a kind of flavonoid, antioxidants in food that scavange free radicals that can damage cells. The consumption of flavanols - the predominant pigment in wine, tea and cocoa - has been associated with a lower risk of heart disease and stroke, and improved blood pressure.

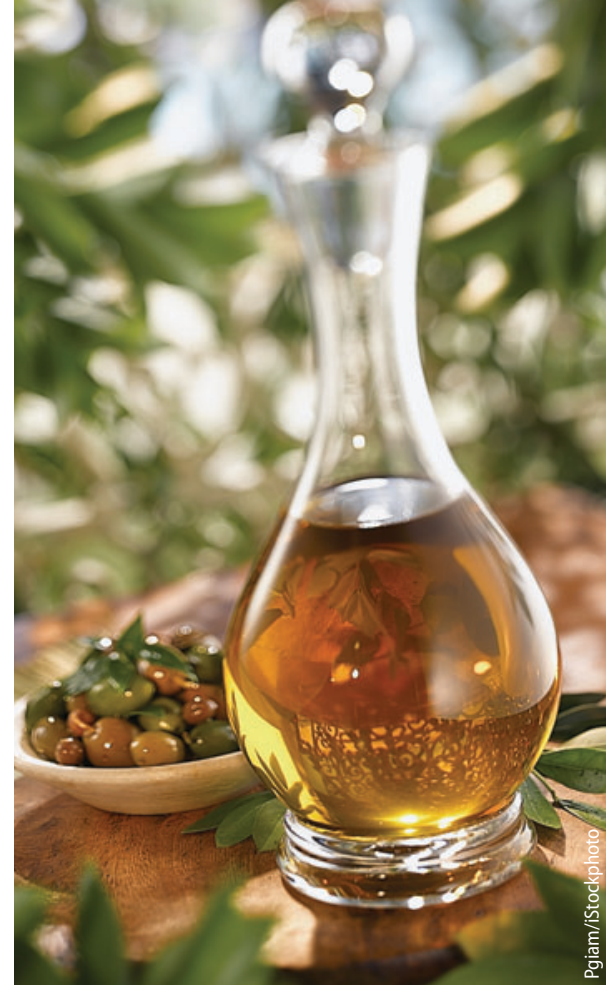

Extra-virgin olive oil contains hydroxytyrosol, a biofactor associated with the healthful effects of a Mediterranean diet. Hydroxytyrosol has been shown to stimulate mitochondrial function.

\section{Flavanols and health}

Flavanols are another subclass of flavonoids and, like quercetin, can exist in a variety of chemical forms and derivatives (Rice-Evans and Packer 2003). Common flavanols include epicatechin and catechin, as well as their oligomers (polymeric chains of these compounds) that are often called proanthocyanidins or procyanidins. They are the predominant pigments in wine, tea and cocoa. The daily estimated intake of flavanols (both monomeric and polymeric forms) is higher than that for flavonols (i.e., compounds similar to quercetin) (fig. 1), amounting to 100 to 300 milligrams per day.

Despite relatively low amounts of catechin and epicatechin in foods, once absorbed and metabolized they have the potential to affect numerous important health parameters (Keen et al. 2005). The consumption of flavanol-rich cocoa and chocolate has been associated with the decreased risk of some vascular diseases, including cardiac events and strokes. Likewise, the daily consumption of foods containing flavanols has been associated with improvements in blood pressure (Desch et al. 2010; Janszky et al. 2009).

In the diet, the smaller or less polymerized forms of flavonoids (monomers and dimers) are more bioavailable than their larger oligomeric counterparts, such as tannins and procyanidins. Studies have 
shown that differences in the bioavailability of bioactive flavonoids are somewhat independent of the food matrix: monomer uptake is largely unaffected by the average diet, and consuming flavanols in water versus milk does not markedly affect their uptake and metabolism (Keen et al. 2005; Schramm et al. 2003).

Flavanols such as epicatechin are thought to influence mitochondrial function through both activation and control of programmed cell death (apoptosis). Epigallocatechin-3-gallate functions as an inhibitor of certain nuclear proteins important to the control (rate) of mitochondriogenesis and apoptosis.

While a good balance is apparently maintained between improved mitochondrial efficiency and orderly apoptosis in normal cells, epicatechins such as epigallocatechin-3-gallate may tip the balance toward apoptosis in certain cancer cell lines (Li et al. 2009). In addition, epigallocatechin-3-gallate has the potential to improve oxidative metabolism (glucose and fatty acid). Low concentrations of epigallocatechin-3-gallate (micromoles) both inhibit glucose production and reduce the expression of genes critical to producing glucose, increasing lipid oxidation and improving insulin sensitivity. At even lower concentrations (nanomoles), epigallocatechin-3-gallate is able to activate 5'-AMP-activated protein kinase (AMPK), a signaling factor that sets the stage for improved oxidative metabolism and fatty acid oxidation.

Generalizing from observations involving epigallocatechin-3-gallate, flavanols have the potential to improve oxidative metabolism as well as accelerate apoptosis in some lines of oncogenic (tumor-producing) cells.

\section{Sources of hydroxytyrosol}

Olive oil. The Mediterranean diet has been associated with a lower incidence of cardiovascular disease and certain cancers (Ordovas et al. 2007; Ortega 2006). Extra-virgin olive oil (produced by only physical and no chemical processing) is the principal source of fat in the Mediterranean diet and has been identified as an important contributor to the reduced mortality associated with this diet. Hydroxytyrosol, a phenolic constituent of olive oil, is considered one of the biofactors associated with the healthful effects

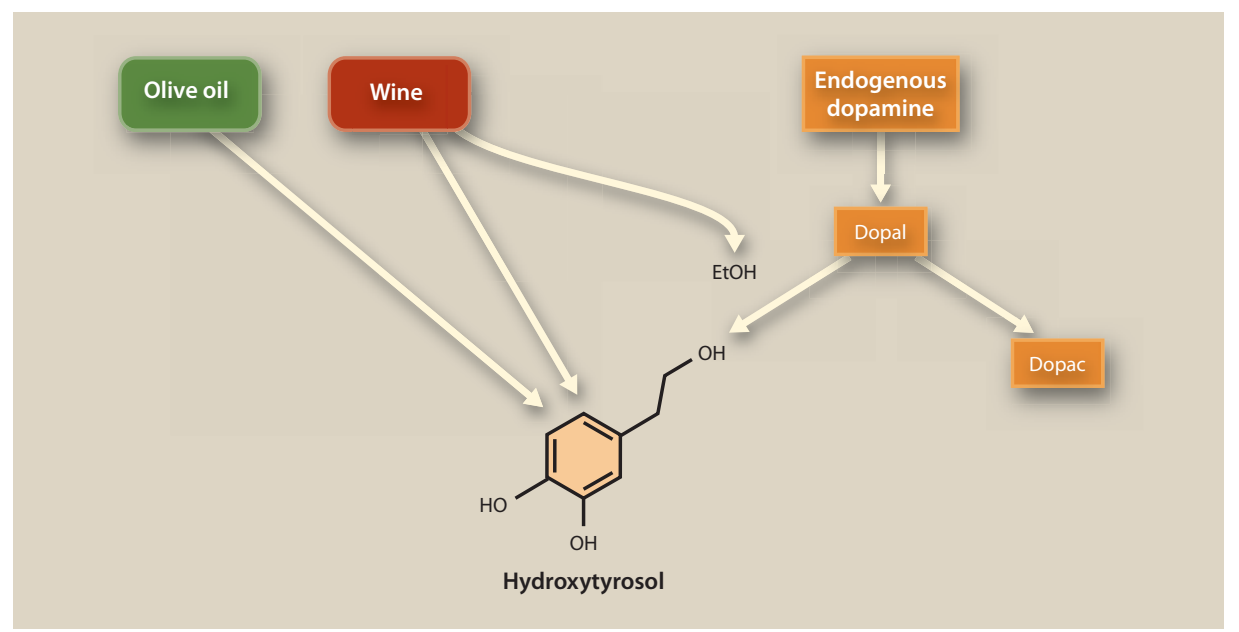

Fig. 2. Sources of the mitochondrial nutrient hydroxytyrosol associated with the Mediterranean diet. Primary nutritional sources are extra-virgin olive oil and wine. The alcohol in wine or distilled spirits may suppress the conversion of endogenous dopamine to dopac (3,4-dihydroxyphenylacetic acid) and redirect it, via a reductive pathway, to dopal (3,4-dihydroxyphenylacetaldehyde) and then to hydroxytyrosol.

of olive oil (Bendini et al. 2007; Bertelli 2007; Fito et al. 2007; Hao et al. 2010).

Indeed, recent research indicates that hydroxytyrosol stimulates mitochondrial function - perhaps constituting a new mechanism by which olive oil lowers the risk of various diseases (Hao et al. 2010). Diabetes and obesity, for which cardiovascular disease is the most common and serious complication, both involve dysfunctional glucose metabolism. Because mitochondrial respiration plays a critical role in glucose metabolism, mitochondrial dysfunction likewise has been shown to be associated with these conditions.

Wine. Although extra-virgin olive oil is the principal source of hydroxytyrosol, this biofactor also occurs in wine - a second hallmark of the Mediterranean diet. Remarkably, it appears that alcohol in wine further boosts hydroxytyrosol levels in the body. A study comparing the effects of wine and olive oil consumption showed that even though the amount of hydroxytyrosol per serving of wine was less than in olive oil (about 0.3 to 0.4 milligrams per 200 to 300 milliliters wine, versus 1.7 milligrams in 50 milliliters oil), bodily amounts as assessed from urinary excretion measurements were $40 \%$ higher than could be attributed independently to either wine or olive oil intake (de la Torre et al. 2006).

This hydroxytyrosol increase, however, could be attributed to an additional effect of the wine. The ethanol in wine (and distilled spirits) apparently affects the pathways that produce the neurotransmitter dopamine, redirecting them to produce hydroxytyrosol instead (fig. 2). In a recent dietary study of 506 males who consumed a diet containing both olive oil and red wine, after adjusting for the contributions of olive oil and wine to hydroxytyrosol, urinary hydroxytyrosol levels increased linearly with alcohol intake (Schöder et al. 2009). In addition to the direct consumption of hydroxytyrosol in wine and olive oil, dietary alcohol can play an indirect role in raising levels of this beneficial biofactor.

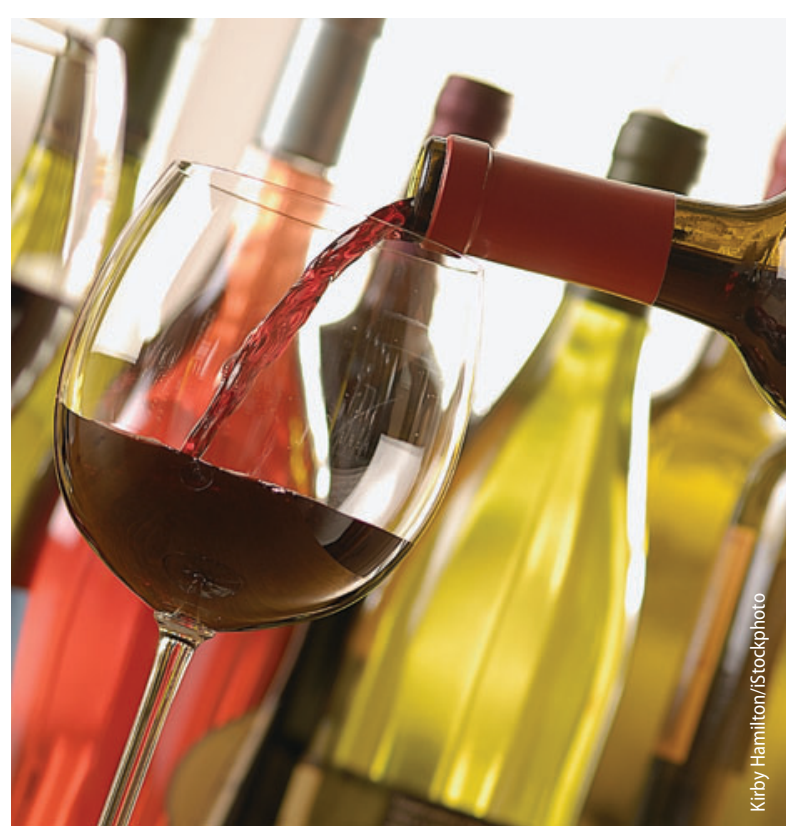

The alcohol in red wine appears to boost hydroxytyrosol levels by redirecting the body to produce hydroxytyrosol instead of dopamine, a neurotransmitter. 
In Mediterranean countries such as Italy and Greece, dietary intakes of olive oil polyphenols are estimated at around 10 to 20 milligrams per day with a daily intake of 25 to 50 milliliters of olive oil. If the olive oil is not chemically treated, about 0.5 to 1 milligram of the polyphenolic fraction is hydroxytyrosol or hydroxytyrosol derivative. A daily serving of one to two glasses of wine could add another 0.3 to 0.4 milligram of hydroxytyrosol (GranadosPrincipal et al. 2010).

PGC-1a and mitochondrial function. Heightened levels of hydroxytyrosol and other mitochondrial enhancers may improve mitochondrial function in two distinct ways. Each involves increasing the expression of a key mitochondriogenesis protein (PGC-1 $\alpha$ ), thereby reducing the risk of developing obesity, diabetes and cardiovascular disease. Increased expression of PGC- $1 \alpha$ also indirectly improves mitochondrial function by interacting with and enhancing enzymes that protect cells against the oxidative damage caused by excessive levels of reactive oxygen species (ROS) (see page 136). For example, increased PGC-1 $\alpha$ protects neuronal cells from oxidative stress. Mice lacking PGC- $1 \alpha$ suffer greater drug-induced oxidative damage to brain and neural tissues (St-Pierre et al. 2006).

\author{
Healthful diets include an abundance of fruits and vegetables \\ that contain these biofactors, providing measurable benefits to \\ mitochondrial health and lessening some disease signs or symptoms.
}

Hydroxytyrosol is thought to be protective because it has the ability to increase PGC-1 $\alpha$ and improve mitochondrial and cellular ROS-related functions. In cultured adipocytes (fat cells), hydroxytyrosol increases both PGC- $1 \alpha$ expression and mitochondrial biogenesis (Hao et al. 2010). Furthermore, hydroxytyrosol has the same beneficial effects on the mitochondria of retinal pigment epithelial cells (derived from the retina) treated with the toxic chemical acrolein, a model for the slowly accumulating damage to the eyes that results in age-related macular degeneration (vision loss). This suggests that hydroxytyrosol may also be useful in treating or delaying age-related macular degeneration (Zhu et al. 2010). In summary, hydroxytyrosol could be potentially therapeutic in preventing and treating type 2 diabetes, obesity and agerelated macular degeneration.

\section{Ubiquity of bacterial PQQ}

In addition to being a plant growth factor, the bacterial compound

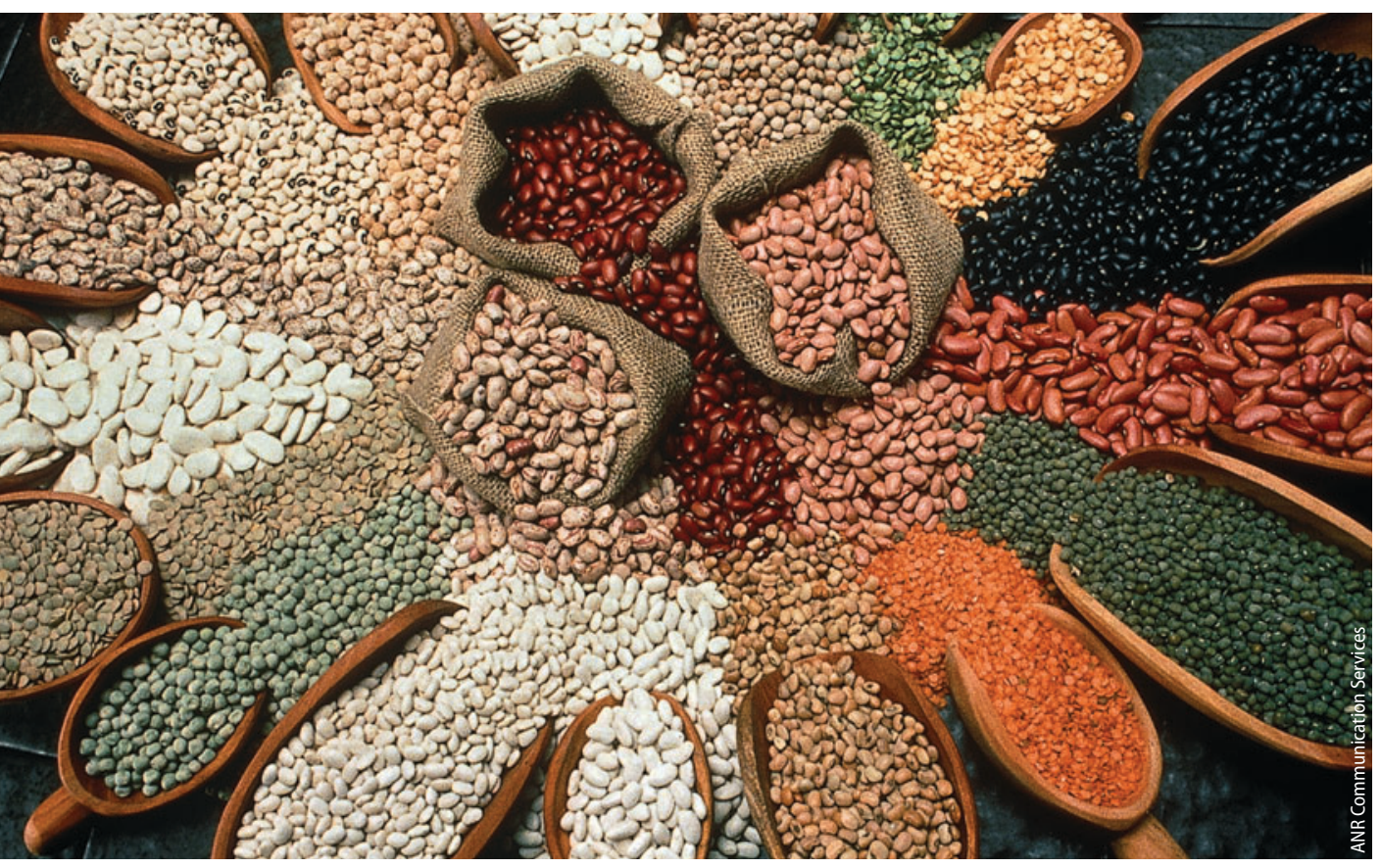

Legumes are an important source of $\mathrm{PQQ}$, a growth factor provided to plants by soil bacteria. Such dietary biofactors stimulate mitochondriogenesis (the conversion of glucose into energy in cells) or inhibit apoptosis (cell death), ultimately improving metabolic health. pyrroloquinoline quinone (PQQ) protects mitochondria from oxidative stress and promotes mitochondriogenesis (Rucker et al. 2009). Many soil bacteria provide plants with PQQ, which increases their height, flower number, fruit number and total fruit weight. Recent evidence suggests that compounds like PQQ are components of interstellar dust (Krueger et al. 2004), considered the precursor of organic materials in early life on the Earth. This ubiquitous presence of PQQ suggests that humans and animals are constantly exposed to this compound. However, although many bacteria make PQQ, most common intestinal bacteria (such as Escherichia coli) do not. Hence, an external source of PQQ may be important in sustaining its levels in human and animal tissues.

PQQ can play a role in pathways important to mitochondrial cell signaling, such as increasing production of the mitochondriogenesis protein PGC- $1 \alpha$ (Chowanadisai et al. 2010; Tchaparian et al. 2010). In addition, PQQ deprivation results in a wide range of systemic responses, including growth impairment, compromised immune responsiveness and abnormal reproductive performance in experimental models. PQQ deprivation also alters lipid and amino acid metabolism, and reverses the effects of mitochondrial oxidation inhibitors.

Dietary sources of PQQ include many fermented products, wine, tea, cocoa and legumes. In animals, deficiency signs are observed at levels at or below 300 micrograms PQQ per kilogram of diet. Human dietary consumption may be estimated at 200 to 400 micrograms PQQ per day, similar to the dietary allowances for vitamins, biotin and folic acid. When mice are fed highly purified diets devoid of PQQ, repletion with 300 micrograms PQQ per kilogram to the diet results in improved growth, mitochondrial function and immune responsiveness. Expressed as a human equivalent, this amounts to 100 to 200 micrograms PQQ per day. In recent unpublished human studies, 10 to 20 milligrams PQQ per day resulted in changes 
in triglyceride levels and measures of antioxidant and anti-inflammatory potential in plasma. However, such amounts are only obtained with supplements and were chosen somewhat arbitrarily. Although about 20 times more than what might be present in the diet, this is nevertheless 20 to 50 times less PQQ than the amounts of quercetin or resveratrol needed to elicit similar effects.

\section{Mitochondria and diet}

Mitochondria are responsible for oxidative metabolism and converting substances from the foods we eat into energy for essential functions. Dietary biofactors that optimize mitochondrial function work by either activating

\section{References}

Agarwal B, Baur JA. 2011. Resveratrol and life extension. Ann NY Acad Sci 1215:138-43.

Balzer JA, RassafT, Heiss C, et al. 2008. Sustained benefits in vascular function through flavanol-containing cocoa in medicated diabetic patients: A double-masked, randomized, controlled trial. J Am Coll Cardio 51:2141-9.

Bass TM, Weinkove D, Houthoofd K, et al. 2007. Effects of resveratrol on lifespan in Drosophila melanogaster and Caenorhabditis elegans. Mech Aging Dev 28:546-52.

Baur JA. 2010. Resveratrol, sirtuins, and the promise of a DR mimetic. Mech Aging Dev 131:261-9.

Baur JA, Pearson KJ, Price NL, et al. 2006. Resveratrol improves health and survival of mice on a high-calorie diet. Nature 444:337-42

Bendini A, Cerretani L, Carrasco-Pancorbo A, et al. 2007. Phenolic molecules in virgin olive oils: A survey of their sensory properties, health effects, antioxidant activity and analytical methods. An overview of the last decade. Molecules 12:1679-719.

Bertelli AA. 2007. Wine, research and cardiovascular disease: Instructions for use. Atherosclerosis 195:242-7.

Chowanadisai W, Bauerly KA, Tchaparian E, et al. 2010. Pyrroloquinoline quinone stimulates mitochondrial biogenesis through CREB phosphorylation and increased PGC-1a expression. J Biol Chem 285:142-52

Cureton KJ, Tomporowski PD, Singhal A, et al. 2009. Dietary quercetin supplementation is not ergogenic in untrained men. J Appl Physiol 107:1095-104.

Davis JM, Carlstedt CJ, Chen S, et al. 2010. The dietary flavonoid quercetin increases VO (2max) and endurance capacity. Int J Sport Nutr Exerc Metab 20:56-62.

Davis JM, Murphy EA, Carmichael MD, Davis B. 2009

Quercetin increases brain and muscle mitochondrial biogenesis and exercise tolerance. Am J Physiol Regul Integr Comp Physiol 296:R1071-7.

de la Torre R, Covas MI, Pujadas MA, et al. 2006. Is dopamine behind the health benefits of red wine? Eur J Nutr 45(5):307-10.

Desch S, Schmidt J, Kobler D, et al. 2010. Effect of cocoa products on blood pressure: Systematic review and meta-analysis. Am J Hypertens 23:97-103.

Dumke CL, Nieman DC, Utter AC, et al. 2009. Quercetin's effect on cycling efficiency and substrate utilization. App Physiol Nutr Metab 34:993-1000

Elliott P, Jirousek M. 2008. Sirtuins: Novel targets for metabolic disease. Curr Opin Investigative Drugs 4:371-8. mitochondriogenesis or inhibiting apoptosis. This in turn improves oxidative defenses and has a major impact on efficient energy utilization by cells. Healthful diets include an abundance of fruits and vegetables that contain these biofactors, providing measurable benefits to $\mathrm{mi}^{-}$ tochondrial health and lessening some disease signs or symptoms; California fruits and vegetables can have an enormous impact on health, ranging from the normalization and control of blood pressure to improving blood lipid and sugar regulation.

\section{S.F. Shenoy is Senior Research Nutritionist,} Pharmavite, Northridge, CA; W. Chowanadisai is Research Associate, UC Davis; E. Sharman is Research Specialist and Biochemist, Department of Neurology, UC Irvine; C.L. Keen is Professor, Department of Nutrition, UC Davis; J. Liu is Professor, Graduate Center for Toxicology, University of Kentucky; and R.B. Rucker is Emeritus Professor, Department of Nutrition, UC Davis.

Robert M. Hackman, Research Nutritionist in the UC Davis Department of Nutrition, served as Guest Associate Editor for this article. We acknowledge the Vitamin Cases Consumer Settlement Fund (Contract No: 06-000427) from the State of California, which provides support for the UC Davis Center for Health and Nutrition Research. In addition, support was received from Project 985, Jiaotong University, Xian, China (J. Liu); Mars, Incorporated (C.L. Keen); and Mitsubishi Gas Chemical Company (R.B. Rucker).
Fito M, de la Torre R, Covas MI. 2007. Olive oil and oxidative stress. Mol Nutr Food Res 51:1215-24.

Granados-Principal S, Quiles 」L, Ramirez-Tortosa CL, et al. 2010. Hydroxytyrosol: From laboratory investigations to future clinical trials. Nutr Rev 68:191-206.

Hao J, Shen W, Yu G, et al. 2010. Hydroxytyrosol promotes mitochondrial biogenesis and mitochondrial function in 3T3-L1 adipocytes. J Nutr Biochem 21(7):634-44.

Hollman PC, de Vries JH, Van Leeuwen, et al.1995. Absorption of dietary quercetin glycosides and quercetin in healthy ileostomy volunteers. Am J Clin Nutr 62:1276-82. Janszky I, Mukamal KJ, Ljung R, et al. 2009. Chocolate consumption and mortality following a first acute myo cardial infarction: The Stockholm Heart Epidemiology Program. J Intern Med 266:248-57.

Kalgaonkar S, Nishioka H, Gross HB, et al. 2010. Bioactivity of a flavanol-rich lychee fruit extract in adipocytes and its effects of oxidant defense and indices of metabolic syndrome in animal models. Phytother Res 24(8):1223-8.

Keen CL, Holt RR, Oteiza Pl, et al. 2005. Cocoa antioxidants and cardiovascular health. Am J Clin Nutr 81:298-3035

Kopp P. 1998. Resveratrol, a phytoestrogen found in red wine: A possible explanation for the conundrum of the "French paradox"? Eur J Endocrinol 138(6):619-20.

Kovacic P, Somanathan R. 2010. Multifaceted approach to resveratrol bioactivity: Focus on antioxidant action, cell signaling and safety. Oxid Med Cell Longev 3:86-100. Krueger FR, Werther W, Kissel J, Er S. 2004. Assignment of quinone derivatives as the main compound class composing "interstellar" grains based on both polarity ions detected by the "Cometary and Interstellar Dust Analyser"(CIDA) onboard the spacecraft STARDUST. Rapid Commun Mass Spectrom 18:103-11.

Lanoue L, Green KK, Kwik-Uribe C, Keen CL. 2010. Dietary factors and risk for acute infant leukemia: Evaluating the effects of cocoa-derived flavanols on DNA topoisomerase activity. Exp Biol Med 235:77-89.

LiW, Nie S, Yu Q Xie M. 2009. (-)- Epigallocatechin-3gallate induces apoptosis of human hepatoma cells by mitochondrial pathways related to reactive oxygen species. J Ag Food Chem 67:6685-91.

Moon HS, Lee HG, Choi YJ, et al. 2007. Proposed mechanisms of (-)-epigallocatechin-3-gallate for anti-obesity. Chem Biol Interact 167:85-98.

Murase T, Haramizu S, Ota N, Hase T. 2009. Suppression of the aging-associated decline in physical performance by a combination of resveratrol intake and habitual exercise in senescence-accelerated mice. Biogerontology $10: 423-34$
Ordovas JM Kaput J, Corella D. 2007. Nutrition in the genomics era: Cardiovascular disease risk and the Mediterranean diet. Mol Nutr Food Res 51:1293-9.

Ortega RM. 2006. Importance of functional foods in the Mediterranean diet. Public Health Nutr 9:1136-40.

Ottaviani Jl, Momma TY, Heiss C, et al. 2011. The stereochemical configuration of flavanols influences the level and metabolism of flavanols in humans and their biological activity in vivo. Free Radic Biol Med 50(2):237-44 Rajaram S, Connell K, Sabate J. 2009. Effect of almondenriched high monounsaturated fat diet on selected markers of inflammation: A randomized, controlled, crossover study. Br J Nutr 103:907-12.

Rice-Evans CA, Packer L (eds.). 2003. Flavonoids in Health and Disease (2nd ed.). New York, NY: CRC Pr. 568 p.

Rucker R, Chowanadisai W, Nakano M. 2009. Potential physiological importance of pyrroloquinoline quinone. Altern Med Rev 14:268-77.

Schramm DD, Karim M, Schrader HR, et al. 2003. Food effects on the absorption and pharmacokinetics of cocoa flavanols. Life Sci 73:857-69.

Schröder H, de la Torre R, Estruch R, et al. 2009. Alcohol consumption is associated with high concentrations of urinary hydroxytyrosol. Am J Clin Nutr 90:1329-35.

Schroeter H, Heiss C, Balzer J, et al. 2006. (-)-Epicatechin mediates beneficial effects of flavanol-rich cocoa on vascular function in humans. Proc Natl Acad Sci USA 103:1024-9

St-Pierre J, Drori S, Uldry M, et al. 2006. Suppression of reactive oxygen species and neurodegeneration by the PGC-1 transcriptional coactivators. Cell 127:397-408.

Tchaparian E, Marshal L, Cutler G, et al. 2010. Identification of transcriptional networks responding to pyrroloquinoline quinone dietary supplementation and their influence on thioredoxin expression, and the JAK/STAT and MAPK pathways. Biochem J 429(3):515-26.

Wallace TC. 2011. Anthocyanins in cardiovascular disease. Adv Nutr 2:1-7.

Xia EQ, Deng GF, Guo YJ, Li HB. 2010. Biological activities of polyphenols from grapes. Int J Mol Sci 11:622-46. Zhu L, Liu Z, Feng Z, et al. 2010. Hydroxytyrosol protects against oxidative damage by simultaneous activation of mitochondrial biogenesis and phase II detoxifying enzyme systems in retinal pigment epithelial cells. J Nutri Biochem 21(11):1089-98. 